\title{
Role of intragrain oxygen diffusion in polycrystalline tin oxide conductivity
}

\author{
C. M. Aldao, ${ }^{1, a)}$ D. A. Mirabella, ${ }^{1}$ M. A. Ponce, ${ }^{1}$ A. Giberti, ${ }^{2}$ and C. Malagù ${ }^{2}$ \\ ${ }^{1}$ Institute of Materials Science and Technology (INTEMA), University of Mar del Plata and National Research \\ Council (CONICET), Juan B. Justo 4302, B7608FDQ Mar del Plata, Argentina \\ ${ }^{2}$ Department of Physics, University of Ferrara, Via Saragat, 1-44100 Ferrara, Italy
}

(Received 2 December 2010; accepted 3 February 2011; published online 29 March 2011)

\begin{abstract}
Resistivity transients of tin oxide films at step isothermal changes in oxygen pressure are investigated. It is expected that, after exposing the samples to oxygen, the resistivity would increase monotonically as barriers become higher to finally reach a plateau at steady state. Here we present experimental results showing a nonmonotonic resistivity transient response that cannot be explained by only considering changes in the Schottky barrier heights. We provide an explanation based on the effects of intragrain oxygen diffusion that accounts for the observed main features of conduction in this polycrystalline material. Oxygen diffuses into the grains annihilating vacancies; the donor concentration is then reduced affecting the sample conductivity. (C) 2011 American Institute of Physics. [doi:10.1063/1.3561375]
\end{abstract}

\section{INTRODUCTION}

It is well known that the electrical properties of polycrystalline ceramic materials are greatly dominated by potential barriers at the grain boundaries. ${ }^{1-3}$ Many technological applications are related to grain boundary phenomena. In particular, due to its low cost and high sensitivity, polycrystalline oxides are used in the detection of diverse gases by simply measuring changes in the film conductivity. ${ }^{4-9}$ As gases chemisorb at grain boundaries, the intergranular potential barriers are modified affecting the film conductivity. ${ }^{10}$

A semiconductor grain boundary becomes electrically active as charge is trapped by intergrain states. These interface states may arise due to the presence of impurity or additive atoms, because of dislocations introduced by the crystallographic mismatch between adjacent grains, or there could be an interface layer which is another oxide. Regardless of the origin, interface states deplete carriers from surrounding grains leading to the formation of a double Schottky barrier. ${ }^{11-13}$ Tin oxide is considered to be an n-type semiconductor exhibiting oxygen vacancies that behave as donor impurities. The interfacial region is relatively thin and the depletion of majority carriers leaves ionized defects (oxygen vacancies in our case) at close grains as a screening charge of the charge accumulated at the interface. ${ }^{10}$ Additional screening could be provided by deep states but we will not include them in this work.

It is well established that the exposure to oxygen decreases the film conductivity while the exposure to reducing gases, such as $\mathrm{CO}$, produce the opposite trend. ${ }^{14}$ In the first case, when oxygen coverage increases, electrons from the bulk are trapped at interfaces, causing that the intergrain barriers become higher. However, we have experimentally observed that, under appropriate conditions, oxygen adsorption affects the film conductivity in an unexpected manner and we proposed that changes in the oxygen vacancy con-

\footnotetext{
a) Author to whom correspondence should be addressed. Electronic mail: cmaldao@mdp.edu.ar.
}

centration within the grains were responsible. ${ }^{4,5,9,15}$ In this work, we present experimental evidence supporting the role of intragrain oxygen diffusion on the electrical conductivity of polycrystalline tin oxide and a model that describes the observed results.

\section{EXPERIMENTAL DETAILS}

Pure $\mathrm{SnO}_{2}$ samples were prepared in two different sessions, obtaining slightly different characteristics. The samples were prepared though a modified sol-gel process described as follows. To a n-butanol solution $0.7 \mathrm{M}$ of tin(II)2-ethylex-anoate (Strem Chemicals) a given amount of deionized water was added drop-wise and the mixture was stirred at room temperature (RT) during $3 \mathrm{~h}$ (the molar ratio of water to $\mathrm{Sn}$ was 4 and the $p \mathrm{H}$ of the solution was adjusted to be 1 with $\mathrm{HNO}_{3}$ ). The resulting gel was dried overnight at $95^{\circ} \mathrm{C}$, giving a yellow powder. This powder was subsequently calcined at $550^{\circ} \mathrm{C}$ for $2 \mathrm{~h}$. Films were prepared starting from pastes obtained by adding, to the above powder, an organic vehicle with a small percentage of glass frit for improving the adhesion of the layers to the substrates. The films were screen-printed onto alumina substrates provided with a heater element at the backside and front interdigital gold contacts. The thickness of the deposition was about 25 $\mu \mathrm{m}$. Samples were then fired for $1 \mathrm{~h}$ at $850^{\circ} \mathrm{C}$ in air (more details can be found in a previous work). ${ }^{16}$ Figure 1 shows SEM images of the samples microstructure. From these images we determined an average grain size of $150 \mathrm{~nm}$ (a) and $60 \mathrm{~nm}$ (b). Films with grains of $150 \mathrm{~nm}$ will be referred hereafter as LG (large grains) and the other as SG (small grains).

Films were placed in a sealed test chamber (designed to contain a single sensor at a time) equipped for flow through technique ${ }^{17}$ which, in turn, were placed in a thermostatic chamber to keep the room temperature at $25^{\circ} \mathrm{C}$. We performed a first experiment using the LG film injecting pure nitrogen at a flow rate of $500 \mathrm{sccm}$ in the sealed chamber until the stabilization of the conductance was reached. Then 

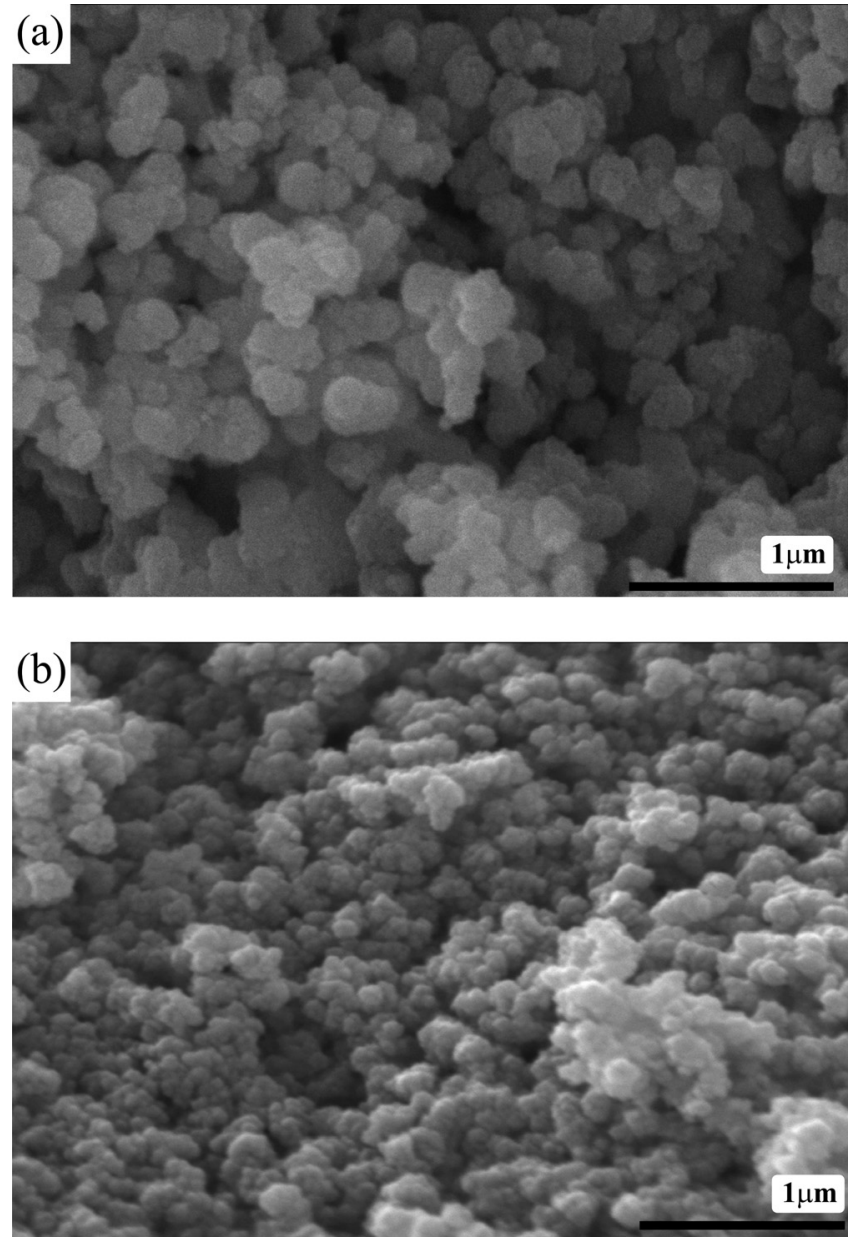

FIG. 1. SEM micrographs of the samples tested. (a) Sample with an average grain size of $150 \mathrm{~nm}$ and (b) sample with an average grain size of $60 \mathrm{~nm}$.

the flow was switched to $500 \mathrm{sccm}$ of synthetic air. The sensor bias was $2.5 \mathrm{~V}$ and conductance variation of the film was measured at 300 and $400{ }^{\circ} \mathrm{C}$. The same experiment was then run on the SG sample.

\section{RESULTS AND DISCUSSION}

Based on the changes in the Schottky barrier heights, it is expected that the resistivity would increase monotonically with time after exposing the samples to oxygen. As oxygen chemisorbs at the intergrains, barriers become higher decreasing the electric conductivity. This behavior has been widely observed in samples with a relatively large grain size and a high density of oxygen vacancies. 5,9,12,18 Oxygen affects intergrain barriers by changing their heights and the widths after adsorption and diffusion into the tin oxide grain take place. First, oxygen is chemisorbed on the surface increasing the barrier height and width; the equilibrium with atmospheric oxygen would be reached rapidly. Second, oxygen diffuses slowly into the grains annihilating vacancies and then reducing the donor concentration. ${ }^{4-6} \mathrm{~A}$ lower donor concentration is responsible for widening the depletion layer even more and accordingly the resistivity increases. We considered that this dependency is a consequence of the relevant role played by tunneling current in polycrystalline materials. ${ }^{19}$
In contrast to the results described above, Fig. 2 shows that samples with grains of $150 \mathrm{~nm}$ width do not show a monotonic increasing of the resistivity as a function of time after the exposition to oxygen. Indeed, a decrease in the resistivity is observed after a quick rise. It had been argued that this behavior could be explained using the fact that, as oxygen diffuses into the grain, depletion regions become wider and Schottky barriers overlap. ${ }^{20}$ Moreover, we found for large enough time (Fig. 2), that the resistance can increase as an evidence that supports the role played by the intragrain oxygen diffusion. Also, in Fig. 2 we show the resistance evolution for small grains that, as for very large grains (not reported in this paper), presents a monotonic increase in the resistivity after oxygen exposure. These results are unexpected and deserve a careful analysis. Below we will introduce a simple one-dimensional model representing the interface between two grains.

In Fig. 3(a) we present a double Schottky barrier model. This description of a semiconductor interface seems to be generally accepted. ${ }^{1,21-23}$ This diagram shows the basic features on an n-type semiconductor junction with and without a voltage applied across the grain boundary. In doing so, we chose to maintain the interface with the same potential; the applied voltage drops part at the left grain, $V_{1}$, and part at the right grain, $V_{2}$. Figure 3(b) shows the charge distribution. Charged donors at both grains generate an electric field and then the band bending. We have assumed that the width of the interfacial region is very small to justify its idealization as an infinitely thin layer with a total charge $-Q_{i}$.

A very important point is to elucidate how the interface charge changes with the applied voltage. Charge neutrality implies that the amount of negative charge at interface states, $Q_{i}$, must be the same than the positive charge at the depletion regions of contiguous grains, $e N_{d}\left(\omega_{1}+\omega_{2}\right)$. Also, the total current flowing from the grains toward the interface must cancel with the total current flowing from the interface toward the grains. We could consider that the conduction

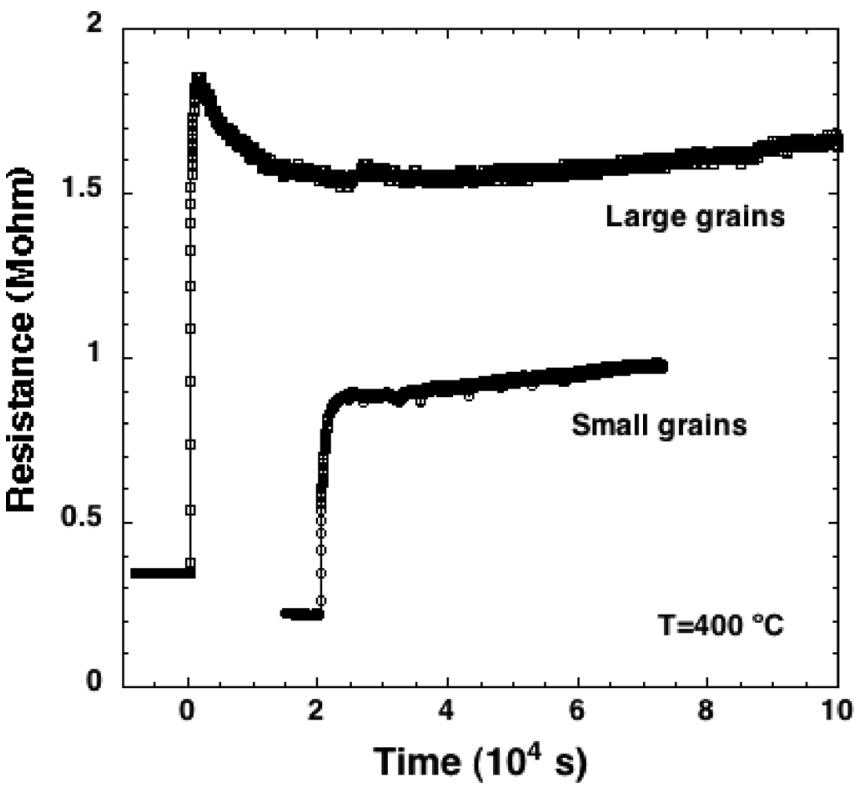

FIG. 2. Resistance versus time curves for samples with large grains, average diameter of $150 \mathrm{~nm}$, and small grains, average diameter of $60 \mathrm{~nm}$. 


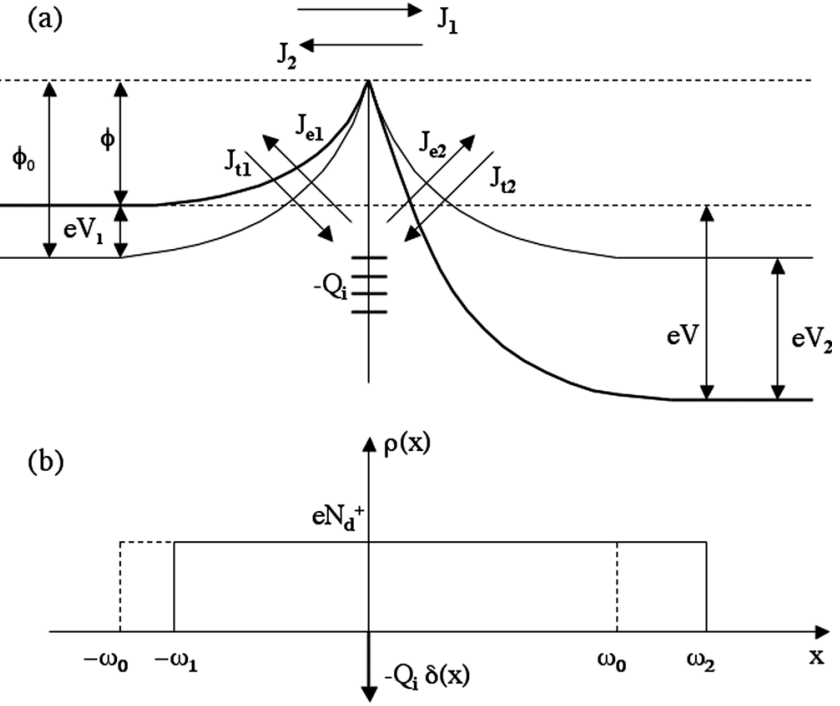

FIG. 3. (a) Energy-band diagram for the double-depletion-layer model. The diagram shows schematically the basic features of an n-type semiconductor interface. Charge trapped at the boundary causes the potential barriers to form. Light line corresponds to the potential when no voltage is applied. Dark line corresponds to the resulting potential after a voltage $V$ was applied across the grain boundary, $V_{1}$ and $V_{2}$ are the applied voltage drops at the left grain and at the right grain, respectively. (b) Charge distribution at the double Schottky barrier. $\omega_{0}$ is the width of the depletion regions when there is no applied voltage; $\omega_{1}$ and $\omega_{2}$ are the widths of the depletion regions when a voltage $V$ is applied to the intergrain.

process occurs as a two stages process, meaning that electrons stop and thermalize at the interface during their passage through it. However, we can adopt a more general approach in which only a fraction of the electrons passing from one grain to the other are captured at the interface.

Electronic transport through a grain interface has two contributions, thermionic and tunnel currents. We will first consider that the carrier transport through the grain boundary occurs only thermionically. Then the electron current density from the negatively biased grain to the positively biased grain is

$$
J_{1}=A T^{2} \exp [-(\phi+\xi) / k T],
$$

where $A$ is the Richardson constant, $T$ is the temperature, $k$ is the Boltzmann constant, and $\xi$ is the difference between the conduction band minimum and the Fermi level. ${ }^{10}$ There is also a current density, $J_{2}$, flowing in the opposite direction of the same form as Eq. (1) but reduced by a factor $\exp (-e V /$ $k T)$. A fraction of these currents, say $c$, is trapped and reemitted by the interface states. The emitted currents must be equal to the trapped currents. The Fermi level in equilibrium, for $V=0$, is constant across the system, so the activation energy for transport from the interface to the grain and vice versa must be $\phi_{0}$. Thus, the emitted currents are

$$
J_{e 1, e 2}=c A T^{2} \exp \left[-\left(\phi_{0}+\xi\right) / k T\right] .
$$

For the sake of simplicity, we will consider that the density of states at the interface is large enough so that the barrier height does not change when the interface charge density increases. ${ }^{24}$ Then, the total trapped current density is

$$
J_{t}=J_{0} \exp (-\phi / k T)[1-\exp (-e V / k T)]
$$

and the total re-emitted current density is

$$
\begin{gathered}
J_{e}=2 J_{0} \exp \left(-\phi_{0} / k T\right), \\
\text { where } J_{0}=c A T^{2} \exp (-\xi / k T) .
\end{gathered}
$$

The current densities $J_{e}=J_{e 1}+J_{e 2}$ and $J_{t}=J_{t 1}+J_{t 2}$ modify the interface charge $Q_{i}$ when the external bias $V$ is changed and determine the voltages $V_{1}$ and $V_{2}$ (see Fig. 3). This model predicts that $\phi$ changes only slightly with the applied voltage. Indeed, for $e V>>k T, \phi_{0}-k T \ln 2$, independent of the applied voltage. Thus, the double Schottky barrier is one sided, with most of the applied voltage dropping on one side.

The above analysis can be easily extended to incorporate tunneling currents. Current densities must be calculated including both thermionic and tunneling contributions. For example, the electron current density from the negatively biased grain to the positively biased grain is

$$
J_{1}=A T^{2} \exp [-(\phi+\xi) / k T]+\frac{A T}{k} \int_{0}^{\phi} F(E) P(E) d E,
$$

where $F(E)$ is the Fermi-Dirac distribution and $P(E)$ is the transmission probability which can be calculated by means of the Wentzel-Kramer-Brilloin (WKB) approximation. $^{21,25,26}$ Similarly, as done above, we can determine the trapped and re-emitted current densities. We have performed these calculations for different applied voltages with $\phi$ as a parameter, so the total trapped and re-emitted current densities are equal. We found that, in general, most of the applied voltage drops at the right side barrier. However, when tunneling currents are taking into account, $V_{1}$ represents a larger fraction of the total applied voltage while $\phi$ reduces slowly with $V$.

Once $\phi$ is known, the total current flowing across the double Schottky barrier can be calculated. A common approach is to consider that most of the current crosses the double barrier in one-step transport process, ${ }^{2}$ so we determined the net current as $J_{1}-J_{2}$ [see Fig. 3(a)].

We have seen that an applied voltage to the double Schottky barrier alters the charge distribution at the interface. Indeed, as it is shown in Fig. 3, the depletion width $\omega_{1}$ reduces and the depletion width $\omega_{2}$ increases. This is a consequence of the availability of free carriers that respond to the applied voltage and contribute to the final potential along the grains. When a voltage (negative on the left, positive on the right) is applied between two grains, electrons rearrange in such a way that the barrier at the left of the interface reduces and the barrier at the right of the interface becomes higher. As discussed above, the barrier on the left (that is directly polarized) does not change much and the larger fraction of the applied voltage drops at the right grain.

When the depletion regions become overlapped, a redistribution of the few available electrons do not contribute significantly. Thus, the resulting potential is simply the sum of the potential at equilibrium and that due to the applied voltage. In Fig. 4 we see the resulting potential. It is obvious that 


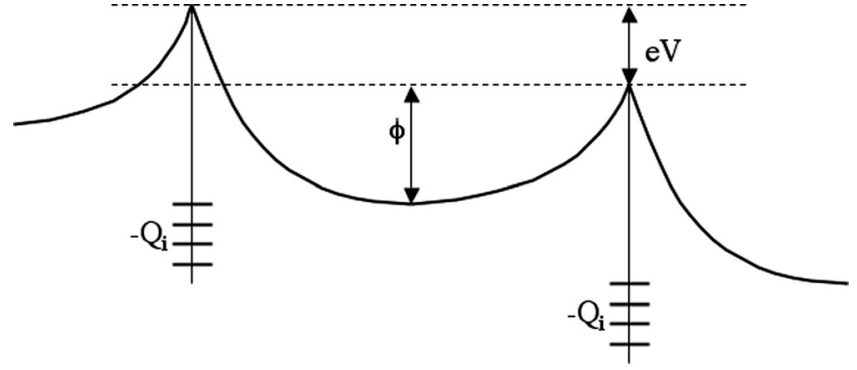

FIG. 4. Energy-band diagram for a depleted grain and its two boundaries with an applied voltage $V$ per grain. Note that, comparing with the band diagram of Fig. 3, the applied voltage directly affects the barrier height $\varphi$.

now the left barrier is reduced by $\sim e V / 2$ and the right barrier is $\sim e V / 2$ higher. This potential facilitates electron transport and then overlapped grains present a lower resistivity.

Several cases for tin oxide intergrains were checked using the models above presented. We used a dopant concentration of $5.6 \times 10^{17} \mathrm{~cm}^{-3}$ because this leads to total depletion of a grain with a diameter of $150 \mathrm{~nm}$. Thus, for nonoverlapped depletion regions, if, for example, $0.066 \mathrm{~V}$ is used as the applied voltage across the grain boundary, the condition that the total trapped and re-emitted current densities be equal determines $V_{1}=0.02 \mathrm{~V}$ and $V_{2}=0.046 \mathrm{~V}$. Once $V_{1}$ and $V_{2}$ are known, we can calculate $J_{1}$ and $J_{2}$ using Eq. (6) and the total current density is found to be $2.57 \times 10^{-7} \mathrm{~A} / \mathrm{cm}^{2}$. If the grains were depleted (model of Fig. 4), the current density is $4.1 \times 10^{-6} \mathrm{~A} / \mathrm{cm}^{2}$ for the same applied voltage, more than an order of magnitude larger than for nondepleted grains. In our experiments, measurements were carried out with an applied voltage of $\sim 1.5 \mathrm{mV}$ per grain. In this case the ratio between overlapped and nonoverlapped intergrain resistivities results to be 13.6. Note that thermionic and tunneling currents were included in these calculations.

For the average grain size of the sample here reported, thermionic and tunneling transport through the grain boundaries have comparable contributions. When the doping is reduced beyond the point at which the depletion layers overlap, the curvature of the potential within the grain reduces. Within the assumptions of our model, the thermionic contribution is not affected. In contrast, the tunneling contribution reduces as the intergranular barrier becomes wider. Thus, after overlapping, our model predicts that eventually, if oxygen diffusion into the grains is high enough, the resistance will increase, as experimentally observed. This final resistance increase cannot be explained resorting to the thermionic current but it comes as a natural consequence of the tunneling current decreasing.

\section{CONCLUSIONS}

Conductivity properties of polycrystalline ceramic materials are generally explained by considering changes in the Schottky barriers heights at the grain boundaries. Based on this mechanism, as oxygen chemisorbs in a polycrystalline tin oxide film, it is expected that the resistivity should increase monotonically as intergranular barriers increase their heights. However, in this work we present experimental results that show a nonmonotonic increase in the resistivity of tin oxide after exposing it to oxygen. We identified the role of intragrain oxygen diffusion as a possible mechanism consistent with the observed experiments and previous studies and developed a simple one-dimensional model that qualitatively accounts for the observed results. The model could be improved by incorporating the three-dimensional character of the problem. Also, we expect that having grains of different sizes would soften the calculated trends and the influence of a finite density of interface states could be calculated. However, in this work, our goal was to report unexpected results and to show that the observed trends can be the consequence of oxygen diffusion into the grains.

This work was partially supported by the ANPCyT (Agencia Nacional de Promoción Científica y Tecnológica, Argentina).

${ }^{1}$ M. Seitz, F. Hampton, and W. Richmond, in Advanced in Ceramics, edited by M. F. Yan and A. H. Heuer (The American Ceramic Society Inc., Ohio, 1983), Vol. 7, pp. 60-70.

${ }^{2}$ M. J. Madou and R. Morrison, Chemical Sensing with Solid State Devices (Academic, San Diego, 1989), p. 6.

${ }^{3}$ P. T. Moseley, Meas. Sci. Technol. 8, 223 (1997).

${ }^{4}$ M. A. Ponce, M. S. Castro, and C. M. Aldao, Mater. Sci. Eng., B 111, 14 (2004).

${ }^{5}$ G. Blaustein, M. S. Castro, and C. M. Aldao, Sens. Actuators B 55, 33 (1999).

${ }^{6}$ M. A. Ponce, C. Malagù, M. C. Carotta, G. Martinelli, and C. M. Aldao, J. Appl. Phys. 104, 054907 (2008).

${ }^{7}$ C. Malagù, V. Guidi, M. C. Carotta, and G. Martinelli, Appl. Phys. Lett. 84(21), 4158 (2004).

${ }^{8}$ X. Wang, S. S. Yee, and W. P. Carey, Sens. Actuators B 24-25, 454 (1995).

${ }^{9}$ M. A. Ponce, C. M. Aldao, and M. S. Castro, J. Eur. Ceram. Soc. 23, 2105 (2003).

${ }^{10}$ Kwan Chi Kao, Dielectric Phenomena in Solids (Elsevier, San Diego, 2004), pp. 327-377.

${ }^{11}$ C. H. Seager and G. E. Pike, Appl. Phys. Lett. 37, 747 (1980).

${ }^{12}$ G. E. Pike, Phys. Rev. B 30, 795 (1984).

${ }^{13}$ G. Blatter and F. Greuter, Phys. Rev. B 33, 3952 (1986)

${ }^{14}$ M. A. Ponce, C. M. Aldao, and M. S. Castro, Mater. Sci. Eng., B 123, 130 (2005).

${ }^{15}$ M. A. Ponce, R. Parra, M. S. Castro, and C. M. Aldao, J. Mater. Sci.: Mater. Electron. Editorial: Springer 18, 1171-1177 (2007).

${ }^{16}$ A. Chiorino, G. Ghiotti, F. Prinetto, M. C. Carotta, D. Gnani, and G. Martinelli, Sens. Actuators B 58, 338 (1999).

${ }^{17}$ A. Giberti, M. C. Carotta, V. Guidi, C. Malagù, G. Martinelli, M. Piga, and B. Vendemiati, Sens. Actuators B 103, 272 (2004).

${ }^{18}$ C. Malagù, M. C. Carotta, G. Martinelli, M. A. Ponce, M. S. Castro, and C. M. Aldao, J. Sens. 2009.

${ }^{19}$ C. Malagù, G. Martinelli, M. A. Ponce, and C. M. Aldao, Appl. Phys. Lett. 92, 162104 (2008).

${ }^{20}$ P. Romppainen and V. Lantto, J. Appl. Phys. 63, 5159 (1988).

${ }^{21}$ C. R. Crowell and V. L. Rideout, Solid-State Electron. 12, 89 (1969).

${ }^{22}$ N. Barsan and U. Weimar, J. Electroceram. 7, 143 (2001).

${ }^{23}$ N. Barsan, D. Koziej, and U. Weimar, Sens. Actuators B 121, 18 (2007).

${ }^{24}$ T. G. G. Maffe[inodot]s, G. T. Owen, C. Malagù, G. Martinelli, M. K. Kennedy, F. E. Kruis, and S. P. Wilks, Surf. Sci. 550, 21 (2004).

${ }^{25}$ M. S. Castro and C. M. Aldao, Appl. Phys. Lett. 63, 1077 (1993).

${ }^{26}$ C. Malagù, M. C. Carotta, A. Giberti, V. Guidi, G. Martinelli, M. A. Ponce, M. S. Castro, and C. M. Aldao, Sens. Actuators B 136, 230 (2009). 